\title{
Information-Theoretic Limits of Dense Underwater Networks
}

\author{
Won-Yong Shin ${ }^{1}$, Daniel E. Lucani ${ }^{2}$, Muriel Médard ${ }^{3}$, Milica Stojanovic ${ }^{4}$, and Vahid Tarokh ${ }^{1}$ \\ ${ }^{1}$ School of Engineering and Applied Sciences, Harvard University, Cambridge, MA 02138, USA \\ ${ }^{2}$ Instituto de Telecommunicacoes, Universidade do Porto, Porto 4200-465, Portugal \\ ${ }^{3}$ Research Laboratory of Electronics, Massachusetts Institute of Technology, Cambridge, MA 02139, USA \\ ${ }^{4}$ ECE Department, Northeastern University, Boston, MA 02115, USA \\ Email: wyshin@seas.harvard.edu; dlucani@fe.up.pt; \{medard, millitsa\}@mit.edu; vahid@seas.harvard.edu
}

\begin{abstract}
Information-theoretic throughput scaling laws are analyzed in an underwater acoustic network with $n$ regularly located nodes on a unit square, in which both bandwidth and received signal power can be severely limited. A narrow-band model is assumed where the carrier frequency is allowed to scale as a function of $n$. We first characterize an attenuation parameter that depends on the frequency scaling as well as the transmission distance. In the dense network having unit area, a cut-set upper bound on the capacity scaling is then derived. We show that there exists either a bandwidth or a power limitation, or both, according to the path-loss attenuation regimes, thus yielding the upper bound that has three fundamentally different operating regimes. In the dense network, we also describe an achievable scheme based on the simple nearest-neighbor multihop transmission. The operating regimes that guarantee the order optimality are identified, where frequency scaling is instrumental towards achieving the order optimality in the regimes.
\end{abstract}

\section{INTRODUCTION}

Gupta and Kumar's pioneering work [1] characterized the connection between the number of nodes $n$ and the sum throughput in large-scale wireless radio networks. They showed that the total throughput scales as $\Theta(\sqrt{n / \log n})$ when a multi-hop ( $\mathrm{MH}$ ) routing strategy is used for $n$ sourcedestination (S-D) pairs randomly distributed in a unit area. ${ }^{1}$ MH schemes are then further developed and analyzed in [2], [3]. A recent result [4] has shown that an almost linear throughput in the network, i.e., $\Theta\left(n^{1-\epsilon}\right)$ for an arbitrarily small $\epsilon>0$, is achievable by using a hierarchical cooperation (HC) strategy. ${ }^{2}$

Along with the studies in terrestrial radio networks, the interest in study of underwater networks has been growing [5], [6], due to recent advances in acoustic communication technology. In underwater acoustic communication systems, both bandwidth and received signal power can be severely limited owing to exponential (rather than polynomial) pathloss attenuation with propagation distance and frequencydependent attenuation. This is a main feature that distinguishes

\footnotetext{
${ }^{1}$ We use the following notation: i) $f(x)=O(g(x))$ means that there exist constants $C$ and $c$ such that $f(x) \leq C g(x)$ for all $x>c$. ii) $f(x)=o(g(x))$ means $\lim _{x \rightarrow \infty} \frac{f(x)}{g(x)}=0$. iii) $f(x)=\Omega(g(x))$ if $g(x)=O(f(x))$. iv) $f(x)=$ $\omega(g(x))$ if $g(x)=o(f(x))$. v) $f(x)=\Theta(g(x))$ if $f(x)=O(g(x))$ and $g(x)=O(f(x))$.

${ }^{2}$ Note that the $\mathrm{HC}$ deals with a subtle issue around quantization, which is not our main concern in this work.
}

underwater systems from wireless radio links. One natural question is what are the fundamental capabilities of underwater networks in supporting multiple S-D pairs over an acoustic channel. To answer this question, the throughput scaling for underwater networks was first studied [7], where $n$ nodes were arbitrarily located in a planar disk of unit area and the carrier frequency was set to a constant. That work showed an upper bound on the throughput of each node, based on the physical model [1], which scales as $n^{-1 / \alpha} e^{-W_{0}\left(\Theta\left(n^{-1 / \alpha}\right)\right)}$, where $\alpha$ corresponds to the spreading factor of the underwater channel and $W_{0}$ represents the branch zero of the Lambert $\mathrm{W}$ function. ${ }^{3}$ Furthermore, a capacity scaling law for extended underwater networks of unit node density was analyzed from an information-theoretic perspective [8]. That work showed both upper and lower bounds on the capacity scaling when the carrier frequency scales as a function of $n$.

In this paper, we analyze a dense underwater network [1], [3], [4], considered as another fundamental network model together with an extended network. ${ }^{4}$ As in [8], we are interested in the case where the carrier frequency scales as a certain function of $n$ in a narrow-band model. Such an assumption leads to a significant change in the scaling behavior owing to the attenuation characteristics. Recently, the optimal capacity scaling of wireless radio networks has been studied in [10] according to operating regimes that are determined by the relationship between the carrier frequency and the number of nodes $n$. The frequency scaling scenario of our study essentially follows the same arguments as those in [10]. We aim to study both an information-theoretic upper bound and achievable rate scaling while allowing the frequency scaling with $n$.

We explicitly characterize an attenuation parameter that depends on the transmission distance and also on the carrier frequency. For networks with $n$ regularly placed nodes, we first

\footnotetext{
${ }^{3}$ The Lambert $\mathrm{W}$ function is defined to be the inverse of the function $z=$ $W(z) e^{W(z)}$ and the branch satisfying $W(z) \geq-1$ is denoted by $W_{0}(z)$.

${ }^{4}$ Capacity scaling laws have intensively been studied in two different networks for analytical convenience: dense and extended networks having unit area and unit node density, respectively. Since the two networks represent both extreme network realizations, a realistic one would be in-between. In wireless radio networks, the work in [9] generalized the results of [4] to the case where the network area can scale polynomially with the number of nodes $n$. In underwater networks, we leave this issue for further study.
} 
derive an upper bound on the total throughput scaling using the cut-set bound. We show that there exists either a bandwidth or a power limitation, or both, according to the operating regimes (i.e., path-loss attenuation regimes), similarly as in Ref. [9]. Specifically, our results indicate that the upper bound has three fundamentally different operating regimes according to the attenuation parameter. In addition, to show constructively our achievability result, we utilize the existing $\mathrm{MH}$ routing scheme with a slight modification, which is suitable for underwater networks due to the very long propagation delay of acoustic signal in water. We identify the operating regimes such that the optimal capacity scaling is guaranteed. We point out that frequency scaling is instrumental towards achieving the order optimality in the regimes.

We refer to the full paper [11] for the detailed description and all the proofs.

\section{System AND Channel MODELS}

We take into account a two-dimensional underwater network that consists of $n$ nodes on a unit square such that two neighboring nodes are $1 / \sqrt{n}$ unit of distance apart from each other, i.e., a regular network [12]. This two-dimensional network is usually assumed to be constituted by sensor nodes that are anchored to the bottom of the ocean. We randomly pick S-D pairings, so that each node is the destination of exactly one source. Each node has an average transmit power constraint $P$ (constant), and we assume that the channel state information is available at all receivers, but not at the transmitters. It is assumed that each node transmits at a rate $T(n) / n$, where $T(n)$ denotes the total throughput of the network.

Now let us turn to channel modeling. We assume frequencyflat channel of bandwidth $W \mathrm{~Hz}$ around carrier frequency $f$, which satisfies $f \gg W$, i.e., narrow-band model. This is a highly simplified model, but nonetheless one that suffices to demonstrate the fundamental mechanisms that govern capacity scaling. Assuming that all the nodes have perfectly directional transmissions, we also disregard multipath propagation. An underwater acoustic channel is characterized by an attenuation that depends on both the distance $r_{k i}$ between nodes $i$ and $k$ $(i, k \in\{1, \cdots, n\})$ and the signal frequency $f$, and is given by

$$
A\left(r_{k i}, f\right)=c_{0} r_{k i}^{\alpha} a(f)^{r_{k i}}
$$

for a constant $c_{0}>0$ independent of $n$, where $\alpha$ is the spreading factor and $a(f)>1$ is the absorption coefficient [5]. The spreading factor describes the geometry of propagation and is typically $1 \leq \alpha \leq 2$. A common empirical model gives $a(f)$ in $\mathrm{dB} / \mathrm{km}$ for $f$ in $\mathrm{kHz}$ as [5]:

$$
10 \log a(f)=a_{0}+a_{1} f^{2}+a_{2} \frac{f^{2}}{b_{1}+f^{2}}+a_{3} \frac{f^{2}}{b_{2}+f^{2}},
$$

where $\left\{a_{0}, \cdots, a_{3}, b_{1}, b_{2}\right\}$ are some positive constants independent of $n$. As stated earlier, we will allow the carrier frequency $f$ to scale at arbitrarily increasing rates relative to $n$. As a consequence, a wider range of both $f$ and $n$ is covered, similarly as in [9], [10]. The absorption $a(f)$ is then an increasing function of $f$ such that

$$
a(f)=\Theta\left(e^{c_{1} f^{2}}\right)
$$

with respect to $f$ for a constant $c_{1}>0$ independent of $n$. The noise $n_{i}$ at node $i \in\{1, \cdots, n\}$ in an acoustic channel can be modeled through four basic sources: turbulence, shipping, waves, and thermal noise [5]. We assume that $n_{i}$ is the circularly symmetric complex additive colored Gaussian noise with zero mean and power spectral density (psd) $N(f)$, and thus $n_{i}$ is frequency-dependent. The overall psd of $n_{i}$ with four sources decays linearly on the logarithmic scale in the frequency region $100 \mathrm{~Hz}-100 \mathrm{kHz}$, which is the operating region used by the majority of acoustic systems, and thus is approximately given by [5]

$$
\log N(f)=a_{4}-a_{5} \log f
$$

for some positive constants $a_{4}$ and $a_{5}$ independent of $n$. It means that $N(f)=O(1)$ since

$$
N(f)=\Theta\left(\frac{1}{f^{a_{5}}}\right)
$$

in terms of $f$ increasing with $n$. The received signal $y_{k}$ at node $k \in\{1, \cdots, n\}$ at a given time is given by

$$
y_{k}=\sum_{i \in I} h_{k i} x_{i}+n_{k}
$$

where

$$
h_{k i}=\frac{e^{j \theta_{k i}}}{\sqrt{A\left(r_{k i}, f\right)}},
$$

represents the complex channel between nodes $i$ and $k, x_{i} \in \mathbb{C}$ is the signal transmitted by node $i$, and $I \subset\{1, \cdots, n\}$ is the set of simultaneously transmitting nodes. The random phases $e^{j \theta_{k i}}$ are uniformly distributed over $[0,2 \pi)$ and independent for different $i, k$, and time. We thus assume a narrowband time-varying channel, whose gain changes to a new independent value for every symbol.

Based on the above channel characteristics, operating regimes of the network are identified according to the following physical parameters: the absorption $a(f)$ and the noise psd $N(f)$ which are exploited here by choosing the frequency $f$ based on $n$. In other words, if the relationship between $f$ and $n$ is specified, then $a(f)$ and $N(f)$ can be given by a certain scaling function of $n$ from (1) and (3), respectively.

\section{CUT-SET UPPER BOUND}

To access the fundamental limit of a dense underwater network, a new cut-set upper bound on the capacity scaling is analyzed from an information-theoretic perspective [13]. Consider a given cut $L$ dividing the network area into two equal halves (see Fig. 1). Under the cut $L$, source nodes are on the left, while all nodes on the right are destinations. In this case, we have an $\Theta(n) \times \Theta(n)$ multiple-input multiple-output (MIMO) channel between the two sets of nodes separated by the cut. 


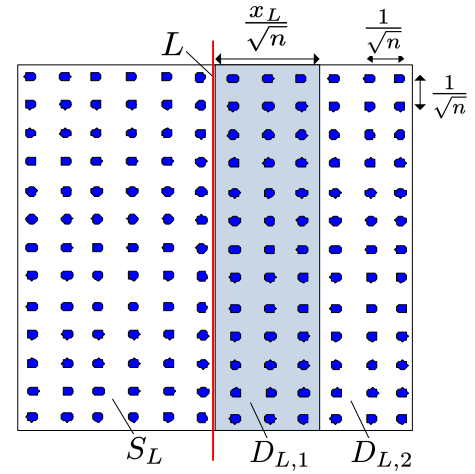

Fig. 1. The cut $L$ in a two-dimensional dense regular network. $S_{L}$ and $D_{L}$ represent the sets of source and destination nodes, respectively, where $D_{L}$ is partitioned into two groups $D_{L, 1}$ and $D_{L, 2}$.

Unlike the extended network case [8], it is necessary to narrow down the class of S-D pairs according to their Euclidean distance to establish a tight upper bound in a dense network. In this section, we use hybrid approaches that consider either the sum of the capacities of the multiple-input single-output (MISO) channel between transmitters and each receiver or the amount of power transferred across the network according to operating regimes, similarly as in Ref. [9].

In the extended network framework [8], upper bounding the capacity by the total received SNR yields a tight bound due to poor power connections for all the operating regimes. In a dense network, however, we may have arbitrarily high received SNR for nodes in the set $D_{L}$ that are located close to the cut, or even for all the nodes, depending on the pathloss attenuation regimes, and thus need the separation between destination nodes that are well- and ill-connected to the lefthalf network in terms of power. More precisely, the set $D_{L}$ of destinations is partitioned into two groups $D_{L, 1}$ and $D_{L, 2}$ according to their location, as illustrated in Fig. 1. Then, by applying generalized Hadamard's inequality, we have

$$
\begin{aligned}
T(n) & \leq E\left[\log \operatorname{det}\left(\mathbf{I}_{\left|D_{L, 1}\right|}+\frac{P}{N(f)} \mathbf{H}_{L, 1} \mathbf{H}_{L, 1}^{H}\right)\right] \\
& +E\left[\log \operatorname{det}\left(\mathbf{I}_{\left|D_{L, 2}\right|}+\frac{P}{N(f)} \mathbf{H}_{L, 2} \mathbf{H}_{L, 2}^{H}\right)\right],
\end{aligned}
$$

where $\mathbf{H}_{L, l}$ is the matrix with entries $\left[\mathbf{H}_{L, l}\right]_{k i}=h_{k i}$ for $i \in S_{L}, k \in D_{L, l}$, and $l=1,2$. Note that the first and second terms in the right-hand side (RHS) of (4) represent the information transfer from $S_{L}$ to $D_{L, 1}$ and from $S_{L}$ to $D_{L, 2}$, respectively. Here, $D_{L, 1}$ denotes the set of destinations located on the rectangular slab of width $x_{L} / \sqrt{n}$ immediately to the right of the centerline (cut), where $x_{L} \in\{0,1, \cdots, \sqrt{n} / 2\}$. The set $D_{L, 2}$ is given by $D_{L} \backslash D_{L, 1}$. It then follows that $\left|D_{L, 1}\right|=x_{L} \sqrt{n}$ and $\left|D_{L, 2}\right|=\left(\sqrt{n} / 2-x_{L}\right) \sqrt{n}$.

Let $T_{l}(n)$ denote the $l$-th term in the RHS of (4) for $l \in$ $\{1,2\}$. It is then reasonable to bound $T_{1}(n)$ by the cardinality of the set $D_{L, 1}$ rather than the total received SNR. In contrast, using the power transfer argument for the term $T_{2}(n)$, as in the extended network case, will lead to a tight upper bound. It is thus important to set the parameter $x_{L}$ according to the attenuation parameter $a(f)$, based on the selection rule for $x_{L}$ [9], so that only $D_{L, 1}$ contains the destination nodes with high received SNR. To be specific, we need to decide whether the total power received by a destination $k \in D_{L}$ from the set $S_{L}$ of sources, denoted by

$$
P_{L}^{(k)}=\frac{P}{c_{0}} \sum_{i \in S_{L}} r_{k i}^{-\alpha} a(f)^{-r_{k i}},
$$

is larger than the noise psd $N(f)$, because degrees-of-freedom (DoFs) of the MISO channel are limited to one. If destination node $k$ has the total received SNR greater than one, i.e., $P_{L}^{(k)}=\omega(N(f))$, then it belongs to $D_{L, 1}$. Otherwise, it follows that $k \in D_{L, 2}$.

For analytical tractability, suppose that

$$
a(f)=\Theta\left(\left(1+\epsilon_{0}\right)^{n^{\beta}}\right) \text { for } \beta \in[0, \infty),
$$

where $\epsilon_{0}>0$ is an arbitrarily small constant, independent of $n$, which represents all the operating regimes with varying $\beta$. For convenience, let us index the node positions such that the source and destination nodes are located at positions $\left(\frac{-i_{x}+1}{\sqrt{n}}, \frac{i_{y}}{\sqrt{n}}\right)$ and $\left(\frac{k_{x}}{\sqrt{n}}, \frac{k_{y}}{\sqrt{n}}\right)$, respectively, for $i_{x}, k_{x}=$ $1, \cdots, \sqrt{n} / 2$ and $i_{y}, k_{y}=1, \cdots, \sqrt{n}$. We then obtain the following scaling results for $P_{L}^{(k)}$ as shown below.

Lemma 1: In a dense network, the term $P_{L}^{(k)}$ in (5) is given by

$$
P_{L}^{(k)}= \begin{cases}O(n) & \text { if } 1 \leq \alpha<2 \text { and } k_{x}=o\left(n^{1 / 2-\beta+\epsilon}\right) \\ O(n \log n) & \text { if } \alpha=2 \text { and } k_{x}=o\left(n^{1 / 2-\beta+\epsilon}\right) \\ O\left(\frac{n^{\alpha / 2}}{\left(1+\epsilon_{0}\right)^{k_{x} n^{\beta-1 / 2}}} \max \left\{1, n^{1 / 2-\beta}\right\}\right) & \text { if } k_{x}=\Omega\left(n^{1 / 2-\beta+\epsilon}\right)\end{cases}
$$

and

$$
P_{L}^{(k)}=\left\{\begin{array}{lr}
\Omega\left(\frac{n^{\alpha / 2-\epsilon}}{k_{x}^{\alpha-1}}\right) & \text { if } k_{x}=o\left(n^{1 / 2-\beta+\epsilon}\right) \\
\Omega\left(\frac{1}{\left(1+\epsilon_{0}\right)^{k_{x} n^{\beta-1 / 2}}} \max \left\{1, \frac{n^{1 / 2-\beta}}{\left(1+\epsilon_{0}\right)^{n^{\beta-1 / 2}}}\right\}\right) & \text { if } k_{x}=\Omega\left(n^{1 / 2-\beta+\epsilon}\right)
\end{array}\right.
$$

for arbitrarily small positive constants $\epsilon$ and $\epsilon_{0}$, where $k_{x} / \sqrt{n}$ is the horizontal coordinate of node $k \in D_{L, 2}$.

Although the upper and lower bounds for $P_{L}^{(k)}$ are not identical to each other, showing these scaling results is enough to make a decision on $x_{L}$ according to the operating regimes. When $k_{x}=o\left(n^{1 / 2-\beta+\epsilon}\right)$, it follows that $P_{L}^{(k)}=\omega\left(n^{\alpha \beta}\right)$ from (8), resulting in $P_{L}^{(k)}=\omega(N(f))$ due to $N(f)=O(1)$. In contrast, under the condition $k_{x}=\Omega\left(n^{1 / 2-\beta+\epsilon}\right)$, it is observed from (7) that $P_{L}^{(k)}$ is exponentially decaying with a function of $n$, thus leading to $P_{L}^{(k)}=o(N(f))$. Consequently, using the result of Lemma 1 , three different regimes are identified and the selected $x_{L}$ is specified accordingly:

$$
x_{L}= \begin{cases}\sqrt{n} / 2 & \text { if } \beta=0 \\ n^{1 / 2-\beta+\epsilon} & \text { if } 0<\beta \leq 1 / 2 \\ 0 & \text { if } \beta>1 / 2\end{cases}
$$

for an arbitrarily small $\epsilon>0$. It is now possible to show the proposed cut-set upper bound in dense networks. 


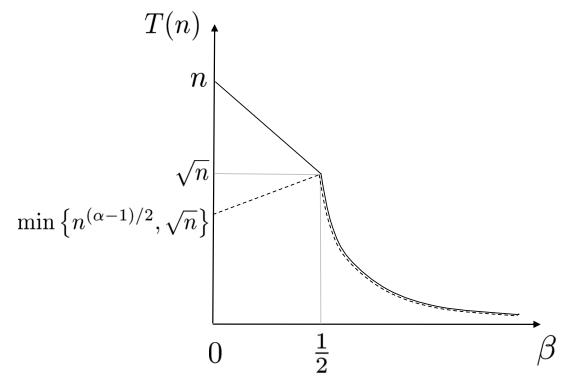

Fig. 2. Upper (solid) and lower (dashed) bounds on the capacity scaling $T(n)$.

Theorem 1: Consider an underwater regular network of unit area. Then, the upper bound on the total throughput $T(n)$ is given by

$$
T(n)= \begin{cases}O(n \log n) & \text { if } \beta=0 \\ O\left(n^{1-\beta+\epsilon} \log n\right) & \text { if } 0<\beta \leq 1 / 2 \\ O\left(\frac{n^{\left(1+\alpha+\beta a_{5}\right) / 2}}{\left(1+\epsilon_{0}\right)^{n^{\beta-1 / 2}}}\right) & \text { if } \beta>1 / 2\end{cases}
$$

where $\epsilon$ and $\epsilon_{0}$ are arbitrarily small positive constants, and $a_{5}$ and $\beta$ are defined in (2) and (6), respectively.

Note that the upper bound [4] for wireless radio networks of unit area is given by $O(n \log n)$, which is the same as the case with $\beta=0$ (or equivalently $a(f)=\Theta(1)$ ) in the dense underwater network. Now let us discuss the fundamental limits of the network according to three different operating regimes shown in (9).

Remark 1: The upper bound on the total capacity scaling is illustrated in Fig. 2 versus the parameter $\beta$ (logarithmic terms are omitted for convenience). We first address the regime $\beta=0$ (i.e., low path-loss attenuation regime), in which the upper bound on $T(n)$ is active with $x_{L}=\sqrt{n} / 2$, or equivalently $D_{L, 1}=D_{L}$, while $T_{2}(n)=0$. In this case, the total throughput of the network is limited by the DoFs of the $\Theta(n) \times \Theta(n)$ MIMO channel between $S_{L}$ and $D_{L}$, and is roughly linear in the bandwidth, thus resulting in a bandwidth-limited network. Our interest is particularly in the operating regimes for which the network becomes powerlimited as $\beta>0$. In the second regime $0<\beta \leq 1 / 2$ (i.e., medium path-loss attenuation regime), the upper bound on $T(n)$ is dominated by the information transfer from $S_{L}$ to $D_{L, 1}$, that is, the term $T_{1}(n)$ contributes more than $T_{2}(n)$. The total throughput is thus limited by the DoFs of the MIMO channel between $S_{L}$ and $D_{L, 1}$, since more available bandwidth leads to an increment in $T_{1}(n)$. As a consequence, in this regime, the network is both bandwidth- and power-limited. In the third regime $\beta>1 / 2$ (i.e., high path-loss attenuation regime), the upper bound for $T_{2}(n)$ is active with $x_{L}=0$, or equivalently $D_{L, 2}=D_{L}$, while $T_{1}(n)=0$. The information transfer to $D_{L}$ is thus totally limited by the sum of the total received SNR from the left-half network to the destination nodes in $D_{L}$. In other words, in the third regime, the network is limited in power, but not in bandwidth.

Note that the upper bound on $T(n)$ decays polynomially with increasing $\beta$ in the regime $0<\beta \leq 1 / 2$, while it drops off exponentially when $\beta>1 / 2$. In addition, another expression on the total throughput $T(n)$ is summarized as follows.

Remark 2: Using (1) and (3) yields the following expression

$T(n)= \begin{cases}O(n \log n) & \text { if } f=\Theta(1) \\ O\left(\frac{n^{1+\epsilon} \log n}{f^{2}}\right) & \text { if } f=\omega(1) \text { and } f=O\left(n^{1 / 4}\right) \\ O\left(\frac{n^{(1+\alpha) / 2} f^{a_{5}}}{e^{c_{1} f^{2} / \sqrt{n}}}\right) & \text { if } f=\omega\left(n^{1 / 4}\right),\end{cases}$

which represents the upper bound for the operating regimes identified by frequency scaling.

\section{AChievability Result}

In this section, to show the order optimality, we analyze the achievable throughput scaling for dense networks with the existing transmission scheme, commonly used in wireless radio networks. In particular, we identify the operating regimes for which the achievable throughput matches the upper bound shown in Section III.

As in the extended network case [8], the nearest-neighbor $\mathrm{MH}$ routing in [1] is used with a slight modification. The basic procedure of the $\mathrm{MH}$ protocol under our dense regular network is similar to the extended network case, and is briefly described as follows:

- Divide the network into $n$ square routing cells, each of which has the same area.

- Draw a line connecting an S-D pair.

- At each node, use the transmit power of

$$
P \min \left\{1, \frac{a(f)^{1 / \sqrt{n}} N(f)}{n^{\alpha / 2}}\right\} \text {. }
$$

The scheme operates with the full power when $a(f)=$ $\Omega\left(\frac{n^{\alpha \sqrt{n} / 2}}{N(f)^{\sqrt{n}}}\right)$. On the other hand, when $a(f)=o\left(\frac{n^{\alpha \sqrt{n} / 2}}{N(f)^{\sqrt{n}}}\right)$, the transmit power $P a(f)^{1 / \sqrt{n}} N(f) / n^{\alpha / 2}$, which scales slower than $\Theta(1)$, is sufficient so that the received SNR at each node is bounded by 1 (note that having a higher power is unnecessary in terms of throughput improvement).

The amount of interference is now quantified to show the achievable throughput based on $\mathrm{MH}$.

Lemma 2: Suppose that a regular network of unit area uses the nearest-neighbor $\mathrm{MH}$ protocol. Then, the total interference power $P_{I}$ from other simultaneously transmitting nodes, corresponding to the set $I \subset\{1, \cdots, n\}$, is bounded by

$P_{I}= \begin{cases}O\left(\frac{\max \left\{n^{(1 / 2-\beta)(2-\alpha)}, \log n\right\}}{n^{\beta a_{5} / 2}}\right) & \text { if } 0 \leq \beta<1 / 2 \\ O\left(n^{-\beta a_{5} / 2}\right) & \text { if } \beta=1 / 2 \\ O\left(\frac{n^{\alpha / 2}}{\left(1+\epsilon_{0}\right)^{n^{\beta-1 / 2}}}\right) & \text { if } \beta>1 / 2\end{cases}$

for an arbitrarily small $\epsilon_{0}>0$, where $a_{5}$ and $\beta$ are defined in (2) and (6), respectively.

From (1), (3), and (6), we note that when $\beta=1 / 2$, it follows that $P_{I}=O(N(f))$, i.e., $P_{I}$ is upper-bounded by the psd $N(f)$ of noise. Using Lemma 2, a lower bound on the capacity scaling can be derived, and hence the following 
result shows the achievable rates under the $\mathrm{MH}$ protocol in a dense network.

Theorem 2: In an underwater regular network of unit area,

$$
T(n)= \begin{cases}\Omega\left(\frac{\sqrt{n}}{\max \left\{n^{(1 / 2-\beta)(2-\alpha)}, \log n\right\}}\right) & \text { if } 0 \leq \beta<1 / 2 \\ \Omega(\sqrt{n}) & \text { if } \beta=1 / 2 \\ \Omega\left(\frac{n^{\left(1+\alpha+\beta a_{5}\right) / 2}}{\left(1+\epsilon_{0}\right)^{n^{\beta-1 / 2}}}\right) & \text { if } \beta>1 / 2\end{cases}
$$

is achievable.

Note that the achievable throughput [1] for wireless radio networks of unit area using $\mathrm{MH}$ routing is given by $\Omega(\sqrt{n})$, which is the same as the case for which $\beta=1 / 2$ (or equivalently $a(f)=\Theta\left(\left(1+\epsilon_{0}\right)^{\sqrt{n}}\right)$ ) in a dense underwater network. The lower bound on the total throughput $T(n)$ is also shown in Fig. 2 according to the parameter $\beta$. From this result, an interesting observation follows. To be specific, in the regime $0 \leq \beta \leq 1 / 2$, the lower bound on $T(n)$ grows linearly with increasing $\beta$, because the total interference power $P_{I}$ in (10) tends to decrease as $\beta$ increases. In this regime, note that $P_{I}$ scales faster than the received signal power $P_{r}$ from the desired transmitter. Meanwhile, when $\beta>1 / 2$, the lower bound reduces rapidly due to the exponential path-loss attenuation in terms of increasing $\beta$.

In addition, similarly to Section III, another expression on the achievability result is now summarized as in the following.

Remark 3: From (1) and (3), it follows that

$$
T(n)= \begin{cases}\Omega\left(\frac{\sqrt{n}}{\max \left\{e^{c_{1}(2-\alpha) f^{2} / \sqrt{n}}, \log n\right\}}\right) & \text { if } f=\Omega(1) \\ \Omega(\sqrt{n}) & \text { and } f=o\left(n^{1 / 4}\right) \\ \Omega\left(\frac{n^{(1+\alpha) / 2} f^{a_{5}}}{e^{c_{1} f^{2} / \sqrt{n}}}\right) & \text { if } f=\Theta\left(n^{1 / 4}\right)\end{cases}
$$

which represents the lower bound for the operating regimes obtained from the relationship between the carrier frequency $f$ and the number of nodes $n$.

Now let us turn to examining how the upper bound is close the achievable throughput scaling.

Remark 4: Based on Theorems 1 and 2, it is seen that if $\beta \geq 1 / 2$, then the achievable rate of the $\mathrm{MH}$ protocol is close to the upper bound up to $n^{\epsilon}$ for an arbitrarily small $\epsilon>0$ (note that the two bounds are of exactly the same order especially for $\beta>1 / 2$ ). The condition $\beta \geq 1 / 2$ corresponds to the high path-loss attenuation regime, and is equivalent to $a(f)=\Omega\left(\left(1+\epsilon_{0}\right)^{\sqrt{n}}\right)$ or $f=\Omega\left(n^{1 / 4}\right)$. Therefore, the $\mathrm{MH}$ is order-optimal in regular networks of unit area under the aforementioned operating regimes, whereas in extended networks [8], using MH routing results in the order optimality for all the operating regimes.

Finally, we remark that applying the HC strategy [4] does not guarantee the order optimality in the regime $0 \leq \beta<1 / 2$ (i.e., low and medium path-loss attenuation regimes). The primary reason is specified under each operating regime: for the condition $\beta=0$, following the steps similar to those of Lemma 2, it follows that $P_{I}=\omega\left(P_{r}\right)$ at all levels of the hierarchy, thereby resulting in the signal-to-interferenceand-noise ratio that scales as $o(1)$ for each transmission (the details are not shown in this paper). It is thus not possible to achieve a linear throughput scaling. Now let us focus on the case where $0<\beta<1 / 2$. At the top level of the hierarchy, the transmissions between two clusters having distance $O(1)$ become a bottleneck because of the exponential path-loss attenuation with propagation distance. Hence, the achievable throughput of the $\mathrm{HC}$ decays exponentially with respect to $n$, which is significantly lower than that in (11).

\section{CONCLUSION}

Dense underwater acoustic networks were analyzed in terms of capacity scaling. Provided that the frequency $f$ scales relative to the number of nodes $n$, the information-theoretic upper bound and the achievable throughput were obtained as functions of the attenuation parameter $a(f)$. The upper bound was first derived by characterizing three different operating regimes, in which there exists either a bandwidth or a power limitation, or both. In addition, from the achievability result, we proved that the $\mathrm{MH}$ protocol is order-optimal in powerlimited regimes (i.e., the case where $f$ scales faster than or as $\left.n^{1 / 4}\right)$.

\section{REFERENCES}

[1] P. Gupta and P. R. Kumar, "The capacity of wireless networks," IEEE Trans. Inf. Theory, vol. 46, pp. 388-404, Mar. 2000.

[2] M. Franceschetti, O. Dousse, D. N. C. Tse, and P. Thiran, "Closing the gap in the capacity of wireless networks via percolation theory," IEEE Trans. Inf. Theory, vol. 53, pp. 1009-1018, Mar. 2007.

[3] A. El Gamal, J. Mammen, B. Prabhakar, and D. Shah, "Optimal throughput-delay scaling in wireless networks-Part I: The fluid model," IEEE Trans. Inf. Theory, vol. 52, pp. 2568-2592, June 2006.

[4] A. Özgür, O. Lévêque, and D. N. C. Tse, "Hierarchical cooperation achieves optimal capacity scaling in ad hoc networks," IEEE Trans. Inf. Theory, vol. 53, pp. 3549-3572, Oct. 2007.

[5] M. Stojanovic, "On the relationship between capacity and distance in an underwater acoustic communication channel," ACM SIGMOBILE Mobile Computing and Communications Review (MC2R), vol. 11, pp. 34-43, Oct. 2007.

[6] D. E. Lucani, M. Médard, and M. Stojanovic, "Underwater acoustic networks: channel models and network coding based lower bound to transmission power for multicast," IEEE J. Select. Areas Commun., vol. 26, pp. 1708-1719, Dec. 2008.

[7] - - "Capacity scaling laws for underwater networks," in Proc. Asilomar Conf. on Signals, Systems and Computers, Pacific Grove, CA, Oct. 2008, pp. 2125-2129.

[8] W.-Y. Shin, D. E. Lucani, M. Médard, M. Stojanovic, and V. Tarokh, "Multi-hop routing is order-optimal in underwater extended networks," in Proc. IEEE Int. Symp. Inf. Theory (ISIT), Austin, TX, June 2010, pp. 510-514.

[9] A. Özgür, R. Johari, D. N. C. Tse, and O. Lévêque, "Informationtheoretic operating regimes of large wireless networks," IEEE Trans. Inf. Theory, vol. 56, pp. 427-437, Jan. 2010.

[10] A. Özgür, O. Lévêque, and D. N. C. Tse, "Linear capacity scaling in wireless networks: beyond physical limits?" in Proc. Inf. Theory and Applications Workshop (ITA), San Diego, CA, Jan./Feb. 2010, pp. 1-10.

[11] W.-Y. Shin, D. E. Lucani, M. Médard, M. Stojanovic, and V. Tarokh, "On the effects of frequency scaling over capacity scaling in underwater networks - Part II: Dense network model," Springer Wireless Pers. Commun., submitted for publication.

[12] L.-L. Xie and P. R. Kumar, "A network information theory for wireless communication: scaling laws and optimal operation," IEEE Trans. Inf. Theory, vol. 50, pp. 748-767, May 2004.

[13] T. M. Cover and J. A. Thomas, Elements of Information Theory. New York: Wiley, 1991. 